\title{
Dual Resonant Slot Antennas for Wireless Applications
}

\author{
Nader Behdad*, and Kamal Sarabandi \\ Department of Electrical Engineering and Computer Science \\ University of Michigan, Ann Arbor, MI, 48109-2122 \\ behdad@eecs.umich.edu, saraband@eecs.umich.edu
}

\section{Introduction}

Large bandwidth, small size, ease of fabrication, low cost, and compatibility to the rest of the RF front end are desirable factors of antennas for many wireless applications. As miniaturization of personal wireless communication (PWC) devices becomes a necessity in today's competitive markets, antenna designers face the problem of finding the optimum antenna geometry to conserve space and retain the desirable radiation characteristics. Printed antennas, because of their desirable characteristics, are an important category of the antennas used in such systems. Among these antennas, slot antennas have not received as much attention as their microstrip counterparts. These antennas are shown to have great miniaturization potential which is a desirable factor in the design of PWC antennas [1]. Miniaturization, however, decreases the antenna efficiency and available bandwidth. Therefore it is important to find the optimum antenna topology that results in the widest bandwidth and highest radiation efficiency for a given printed circuit board (PCB) area. This necessitates understanding the relation between the geometry of a particular slot antenna and its bandwidth.

Geometrical and physical parameters of the slot as well as the feed network characteristics affect the bandwidth of slot antennas. By considering a slot antenna as an aperture with a known electric field distribution, it is possible to obtain its fields from the Fourier transform of the electric field over the aperture [2]. Using this expression for antenna field, the radiated and stored energies can be found and a quality factor $(Q)$ is defined for the antenna which is a function of the antenna dimensions. It is shown that for a rectangular slot antenna, as the width is increased, the BW increases or equivalently $Q$ decreases. This increase is much faster for the smaller antenna widths and decreases as the width increases beyond a certain range.

This phenomenon is also observed in microstrip fed slot antennas but for these antennas, as the width of the slot increases, another interesting phenomenon occurs. If a narrow microstrip feed is used, at a frequency slightly higher than the original resonant frequency, another resonance occurs which has a similar electric field (magnetic current) distribution with that of the first one. If appropriately designed, the two resonances can be chosen such that the overall bandwidth of the antenna is increased or a dual band antenna is obtained. In this paper, first a brief study on bandwidths of slot antennas is presented and then the design and measurement results of the dual resonant slot antenna is presented and discussed.

\section{Bandwidths of Single Resonant Slot Antennas}

The radiated fields of a slot antenna in a ground plane can be obtained by knowing the electric field distribution over the aperture from the following equation [2]:

$$
E(x, y, z)=\frac{1}{4 \pi^{2}} \iint \tilde{E}\left(k_{x}, k_{y}, z\right) e^{-j k_{x} x} e^{-j k_{y} y} e^{-j k_{z} z} d k_{x} d k_{y}
$$


where $\tilde{E}\left(\mathbf{k}_{\mathrm{x}}, \mathbf{k}_{\mathrm{y}}, \mathrm{z}\right)$ represents the Fourier transform of the aperture electric field. The contribution of the integrand over the visible region of the spectrum $\left(\mathrm{k}_{\mathrm{x}}{ }^{2}+\mathrm{k}_{\mathrm{y}}{ }^{2}<\mathrm{k}_{0}{ }^{2}\right)$ provides the radiated fields and its contribution over the invisible region $\left(\mathrm{k}_{\mathrm{x}}{ }^{2}+\mathrm{k}_{\mathrm{y}}{ }^{2}>\mathrm{k}_{0}{ }^{2}\right)$ results in the evanescent fields that are stored in the vicinity of the antenna and contribute to the reactive part of the antenna impedance. The integral in (1) represents a continuous spectrum of plane waves each carrying a power density of $\tilde{E}^{2} / \eta$. Total radiated and stored powers can be computed by calculating the integral of this power density over the visible and invisible regions, respectively. It is possible to define a quality factor $(\mathrm{Q})$ as:

$$
Q=\omega \frac{\text { Stored Energy }}{\text { Radiated Power }}(2)
$$

The antenna bandwidth, for single resonance antennas, is considered to be inversely proportional to this quality factor [3]. Having defined a relation between the Fourier transform of the electric field over the antenna aperture and antenna bandwidth, it is important to take the aperture dimensions into account as well. Considering a sinusoidal field distribution over a rectangular slot of the following form:

$$
E(x, y, 0)=\cos \left(\frac{\pi x}{L}\right) P_{W, L}(x)
$$

where $P_{W, L}(x)$ is a two dimensional pulse function with length and width of $\mathrm{L}$ and $\mathrm{W}$, the Fourier transform of the aperture electric field can easily be obtained. Figure 1 shows the normalized amplitude of the two dimensional Fourier transform of (3), which is a multiplication of sinc function in $k_{y}$ domain and $g\left(k_{x}\right)=\cos \left(k_{x} L / 2\right) /\left(k_{x}^{2} L^{2}-\pi^{2}\right)$ in $k_{x}$ domain. The width of the sinc function in $k_{y}$ is inversely proportional to the width of the slot antenna. For smaller $\mathrm{W}$ values the sinc function in the $k_{y}$ domain is very wide; therefore it is expected that by increasing $\mathrm{W}$ and reducing the width of the sinc function, the number of radiating plane waves increases, which results in a decreased $Q$. Thus, the bandwidth of the antenna will be increased by increasing the slot width (W). This derivation is valid for slot antennas without a dielectric substrate. In order to account for the presence of the dielectric, the integral in (1) should be modified accordingly [2]. Nevertheless, dielectric covered slot antennas also show a similar behavior. In order to observe the changes in the bandwidth of microstrip-fed slot antennas on a dielectric substrate, a number of full wave simulations are carried on for antennas with different widths. These antennas are $\lambda 2$ (at $f_{0}=1.3 \mathrm{GHz}$ ) slots on a dielectric substrate with relative permittivity of 3.4 and thickness of $500 \mu \mathrm{m}$ and are fed by an off-centered open-circuited microstrip line where the length of the open-circuited stub is changed to obtain a good match for different values of the slot width (W). The - $10 \mathrm{~dB}$ impedance bandwidth of the antenna is recorded as a function of its width (W). Figure 2 shows the percentage bandwidth of these antennas as a function of W. As expected, it is shown that the antenna bandwidth increases as the width of the antenna is increased. However, for $W>0.07 \lambda_{0}$ the double resonant behavior mentioned in section $I$ is observed and can be exploited to drastically increase the antenna bandwidth.

\section{Double Resonant Slot Antennas with Enhanced Bandwidth}

If the slot antenna is fed near one edge by a narrow microstrip line at a proper location and for a proper slot width, at a frequency above that of the first resonance, a fictitious short circuit near the microstrip feed may be created. Basically the tangential electric field created by the narrow microstrip line at a particular distance cancels out the electric field of the slot excited by the return current of the microstrip line on the ground plane. That is, electromagnetically a second short circuit is created for which there is a second resonant frequency. A full wave simulation shows the field distribution for this situation 
in Figure 3(b) whereas Figure 3(a) shows the field distribution at the first (normal) resonant frequency of the slot. The width of the microstrip feed and the distance between the feed and the edge of the slot antenna, $L_{S}$, are two parameters that affect the existence and frequency of this fictitious short circuit. As $\mathrm{L}_{\mathrm{s}}$ increases, the second resonant frequency also increases therefore by choosing $L_{s}$ properly, the frequency of the second resonance can be chosen such that antenna bandwidth is increased or a dual band operation is achieved. As can be observed from Figure 3, the electric field distribution at the second resonant frequency is similar to that of the first one; therefore, it is expected that the radiation patterns of the antenna at the two frequencies are similar.

In order to confirm this phenomenon, two dual-resonant slot antennas with different $\mathrm{L}_{\mathrm{s}}$ values were designed and fabricated on a $500 \mu \mathrm{m}$ thick, $\mathrm{RO} 4350 \mathrm{~B}$ substrate with dielectric constant of 3.4 and loss tangent of 0.003 . Figure 4 shows simulated and measured return losses of these antennas and Table I shows a summary of radiation parameters and physical dimensions of these two antennas. The slight discrepancy between the simulation and measurements can be attributed to the finiteness of the ground plane which causes a shift in the resonance frequency.

The radiation patterns of the Antenna 1 were measured in the anechoic chamber of the University of Michigan at the two resonant frequencies. Figures $5 \mathrm{a}$ and $5 \mathrm{~b}$ show the $\mathrm{E}$ and H-plane co- and cross-polarized patterns at $3.077 \mathrm{GHz}$ and $3.790 \mathrm{GHz}$ respectively. It is shown that the patterns at both resonant frequencies are similar to each other confirming the fact that at both resonant frequencies, the field distributions are similar.

The antenna gains at the two resonant frequencies were also measured in the anechoic chamber using a standard double ridged horn antenna and are reported in Table I.

\begin{tabular}{|l|l|l|l|l|}
\hline Type & $\mathrm{L}, \mathrm{W}$ & $\mathrm{Ls}, \mathrm{Lm}$ & Bandwidth & Gain $@ \mathrm{f}_{b} f_{u}$ \\
\hline Antenna 1 & 37,6 & $3.6,4.1$ & $30.3 \%$ & $2.5 \mathrm{~dB}, 2.6 \mathrm{~dB}$ \\
\hline Antenna 2 & 37,6 & $4.6,3.9$ & $37.0 \%$ & $2.5 \mathrm{~dB}, 2.7 \mathrm{~dB}$ \\
\hline
\end{tabular}

TABLE I. PHYSICAL AND RADIATION PARAMETERS OF THE BROADBAND SLOT

ANTENNA ALL DIMENSIONS ARE IN mm

* Maximum gain in the E-Plane.

\section{Conclusions}

A qualitative relationship between dimensions of rectangular slot antennas and their bandwidths is studied. It is shown that by increasing the width of a rectangular slot antenna, its bandwidth also increases. For appropriately designed microstrip-fed slots, this increase results in creation of a second resonance with similar current distribution with that of the first one and a frequency above the first and below the second normal resonant frequencies. This phenomenon is exploited to achieve an antenna with large bandwidth and similar radiation patterns across the entire band.

\section{References}

[1] Behdad, N., and K. Sarabandi, "Slot Antenna Miniaturization Using Distributed Inductive Loading", IEEE Antennas and Propagation Symposium, Columbus, OH, June 22-27 2003.

[2] Balanis, C. A., “Antenna Theory: Design and Analysis, Chap. 12", Wily, $2^{\text {nd }}$ Ed., 1996.

[3] Geyi, W., P. Jarmuszewski, and Y. Qi, "The Foster Reactance Theorem for Antennas and Radiation Q", IEEE Trans. Antennas and Prop. Vol. 48, 2000, pp. 401-408 

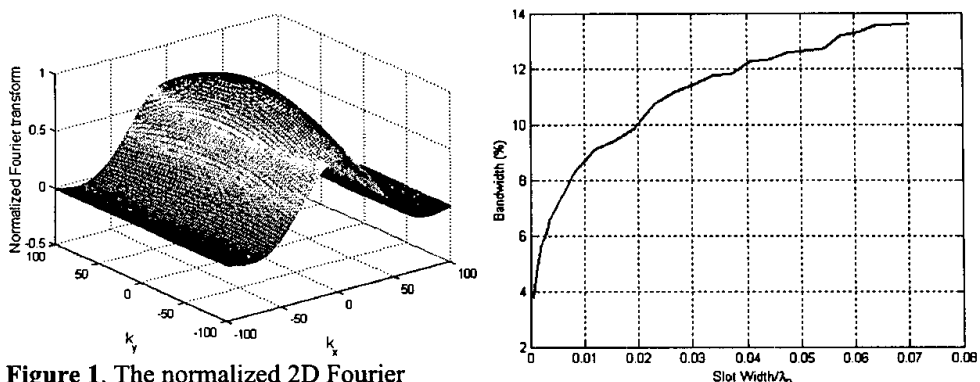

Figure 1. The normalized 2D Fourier transform of a sinusoidal electric field distribution over the aperture.

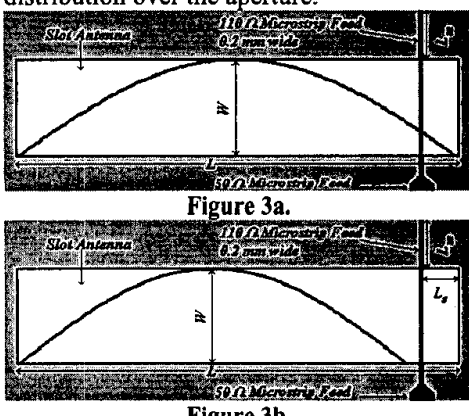

Figure 2. Bandwidths of a $\lambda / 2$ microstripfed slot antenna as a function of its width.

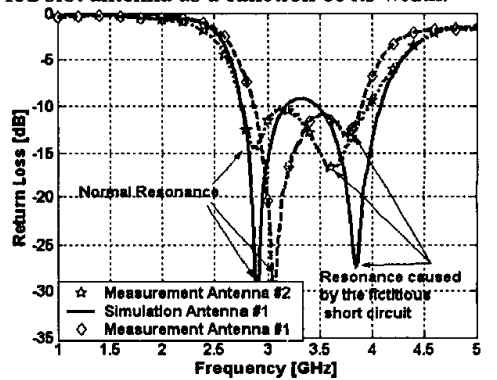
Figure 3b.

Figure 3. Magnetic current distribution along a relatively wide slot antenna at a) the first resonant frequency and $\mathbf{b}$ ) the fictitious second resonant frequency.

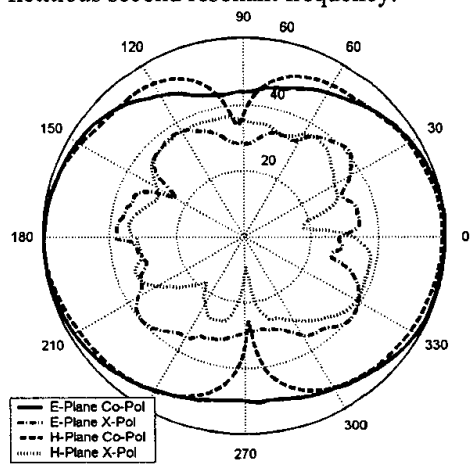

Figure 4. Simulated and measured return losses of the dual-resonant micrsotrip fed slot antennas.

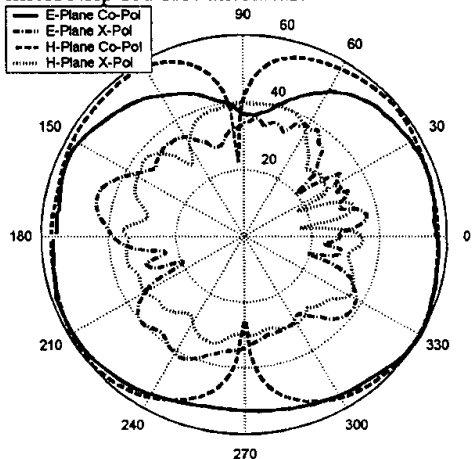

Figure 5b.

Figure 5. Measured radiation patterns of the relatively wide slot antenna at a) $f=3077$ $\mathrm{MHz}$ and b) $f=3790 \mathrm{MHz}$. 